\author{
EVS27 \\ Barcelona, Spain, November 17-20, 2013
}

\title{
Development of Intelligent Electric Vehicle for Study of Unmanned Autonomous Driving Algorithm
}

\author{
In Yub Kim ${ }^{1}$, Kwan Suk Yang ${ }^{1}$, Jin joo Baek ${ }^{1}$, Sung-Ho Hwang ${ }^{1}$ \\ ${ }^{1}$ School of Mechenical Engineering Sungkyunkwan University, Suwon 440-746, Gyeonggi, Korea, hsh@me.skku.ac.kr \\ ${ }^{2}$ School of Mechatronics Engineering Sungkyunkwan University, Suwon 440-746, Gyeonggi, Korea
}

\begin{abstract}
World-class automakers such as BMW, Audi, GM and even IT Company Google are giving much effort in developing unmanned autonomous vehicles. As it can be seen from IEEE report that 3 of every 4 vehicles in 2040 will be unmanned, the studies on future unmanned vehicles have become very important issues these days. This report proposes an intelligent electric vehicle, developed for unmanned autonomous driving algorithm study. This intelligent electric vehicle directly controls traditional steering device and acceleration/brake pedals through electrical signals with electronic actuators which were equipped at each component. Also, various sensors were utilized to recognize surrounding situations, obstacles, and road boundary instead of human. The obtained sensor data and vehicle state are used for performing of unmanned autonomous driving, evaluating the performance of intelligent vehicles.
\end{abstract}

Keywords: Electric Drive, EV, Intelligent, NEV (neighbourhood EV)

\section{Introduction}

Future automobile market will be led by echofriendly, highly safe automobile technology. The fuel efficiency and safety of the vehicle have depended on the driving habit of the driver, but once the current ADAS (Advanced Driving Assist System) technology is commercialized, furthermore, as the unmanned autonomous driving vehicle technology advances, the vehicle will control itself to prevent traffic accidents and enable high-efficiency echo-friendly driving.

To commercialize unmanned autonomous vehicle technology, technologies from various areas should be integrated. Various sensors should be able to recognize the circumstances around the vehicle, and based on this information, the vehicle should be able to control itself for a safe driving to the destination. Also, since the sensors used for these unmanned autonomous driving vehicles are very expensive, it is essential to design a system that can minimize the number of sensors by using the sensors appropriately and organizing efficiently. This report proposes the intelligent electric vehicle platform, developed for researching unmanned autonomous driving vehicle technology. And unmanned autonomous driving algorithm is implemented to verify the performance of this intelligent electric vehicle platform.

\section{Vehicle Platform Composition}

\subsection{Concept and Design}

Table 1 shows the specification of the electrical vehicle platform for unmanned autonomous driving. The driving motor is $7 \mathrm{~kW}$ Induction Motor, and the power source is $72 \mathrm{~V}$ battery pack. For motor controller, Gen4 model of SEVCON was used. 
Table 1 : Vehicle Specification

\begin{tabular}{|l|l|}
\hline Length & $2880 \mathrm{~mm}$ \\
\hline Width & $1683 \mathrm{~mm}$ \\
\hline Height & $1450 \mathrm{~mm}$ \\
\hline Wheel Base & $2150 \mathrm{~mm}$ \\
\hline Tread (Front) & $1420 \mathrm{~mm}$ \\
\hline Tread (Rear) & $1420 \mathrm{~mm}$ \\
\hline Weight & $980 \mathrm{~kg}$ \\
\hline Top Speed & $40 \mathrm{kph}$ \\
\hline Driving Range & $60 \mathrm{~km}$ \\
\hline Traction Motor & $7 \mathrm{~kW}$ Induction type \\
\hline Battery & $72 \mathrm{~V}$ Battery pack \\
\hline
\end{tabular}

Figure 2 shows the concept design for initial unmanned vehicle platform development. In case of common unmanned vehicles, the driveroperated components were indirectly controlled by using mechanical devices for the unmanned driving, but this vehicle is a platform only for unmanned autonomous driving. Brake and acceleration pedal and steering wheel has been removed to secure over $10 \%$ of space compared to conventional vehicles. Each actuator can be controlled with signals.

\subsection{Concept and Design}

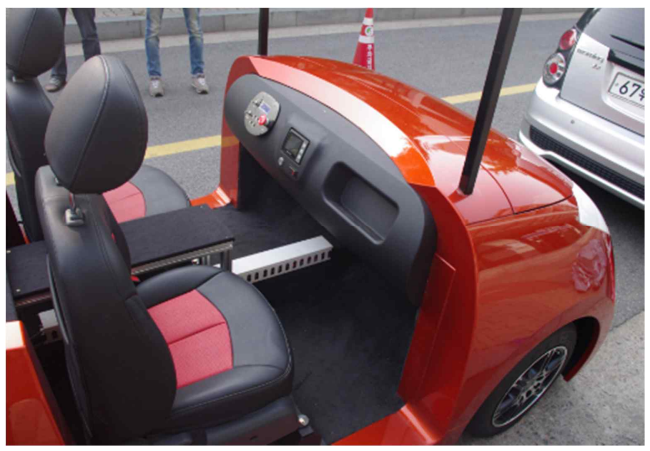

Figure 1: Sungkyunkwan University's UEV

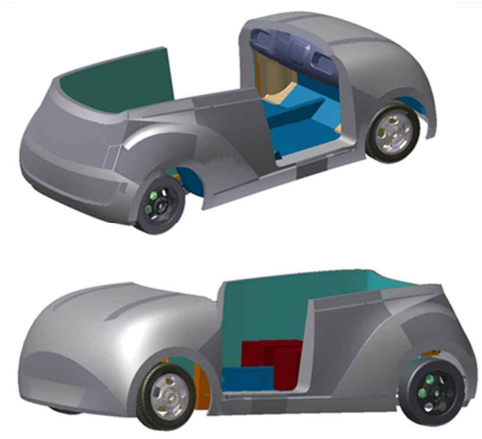

Figure 2: Conceptual Design

\subsubsection{VCU System Architecture}

VCU is the controller that controls the vehicle behavior, and Single board Rio of NI was loaded. It carries RS232c and CAN communication module, and has Digital I/O and Analog I/O channels. VCU controls each actuator and subordinate controller according to the longitudinal/lateral orders form PCU (Perception Control Unit), which estimates the vehicle state and plans path Also, it sends feedback of vehicle operation state to PCU.

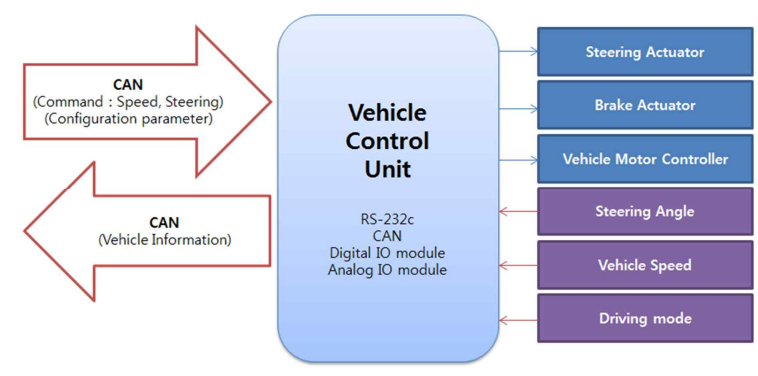

Figure 3: VCU System Architecture

\subsubsection{Steering Control}

For steering control, Steering module of electric power steering type was used. $24 \mathrm{~V} 420 \mathrm{~W}$ servo motor was used for operation, and EPS can be controlled by RS-232c. Also, it has a sensor that can measure the angle of steering wheel, which can be communicated through CAN.

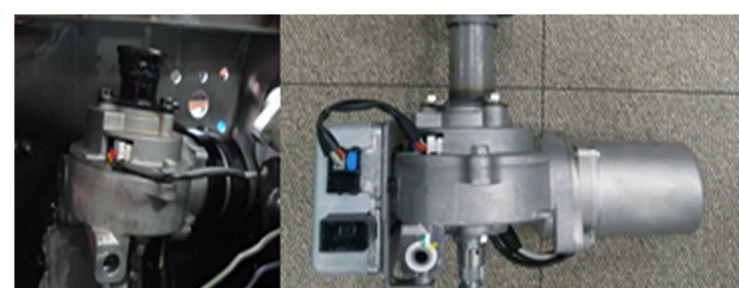

Figure 4: Steering System using EPS

\subsubsection{Brake Control}

For brake control, linear actuator was designed in front of the hydraulic cylinder. 24V 200W SMART motor of Moog Animatics was used, and 60:1 decelerator was connected to the servo motor output axis to deliver sufficient torque to the brake cylinder. Screw-type slider of link structure and universal joint was used to deliver brake torque in the design. The brake servo motor also can be controlled through CAN communication. Effective stroke length is $2 \mathrm{~cm}$. 


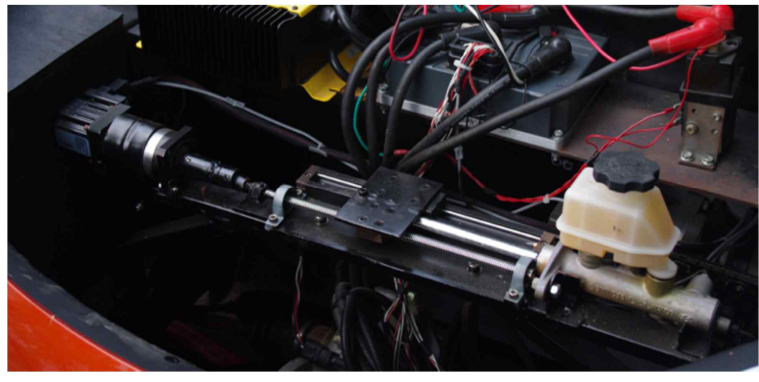

Figure 5: Brake Actuator System

\subsubsection{Acceleration Control}

Acceleration control was implemented by sending an electrical signal directly to the motor controller instead of the existing acceleration pedal. Analog voltage between $0-5 \mathrm{~V}$ is applied to control the operation of traction motor. Also, CAN communication terminal of motor controller is used for real-time feedback of current motor RPM information. This was used for longitudinal control of the intelligent electric vehicle.

\subsection{Sensor and PCU for Unmanned Autonomous Driving}

\subsubsection{Sensor Fusion System Architecture}

Various sensors are used for unmanned autonomous driving, and there is much difference in the performance of unmanned vehicle depending on how the low data from sensors are utilized. Figure 6. Shows the architecture of surrounding environment recognition controller and sensors used in intelligent electric vehicle. PXI system of NI was used to construct this, and LIDAR sensors, DGPS, Vision Camera and AHRS sensor were used.

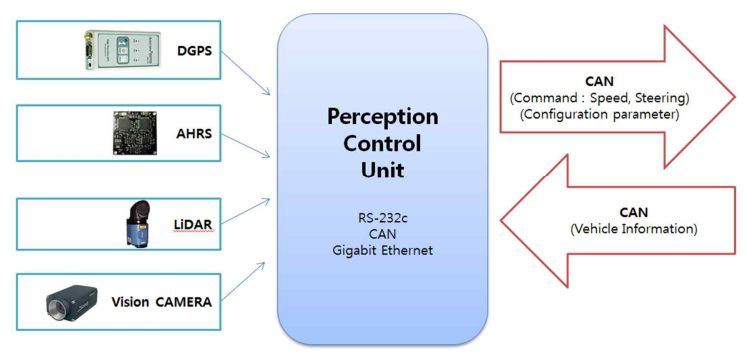

Figure 6: PCU System Architecture

As in Figure 7, it is necessary to have each algorithm to overcome various circumstances for performing of unmanned autonomous driving;

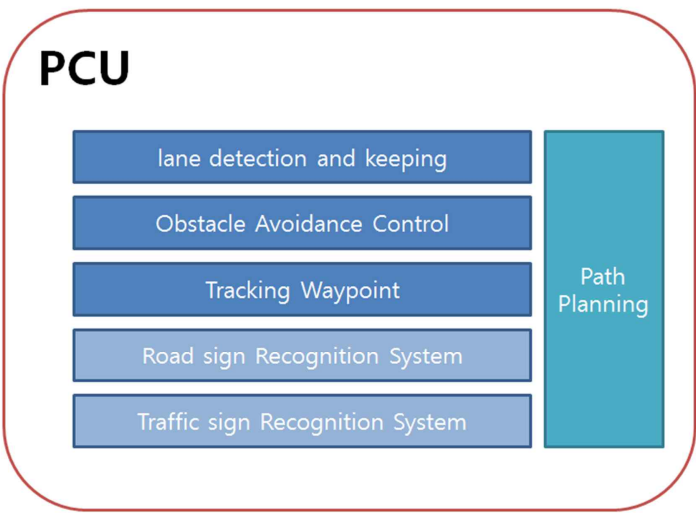

Figure 7 Autonomous Driving Algorithms

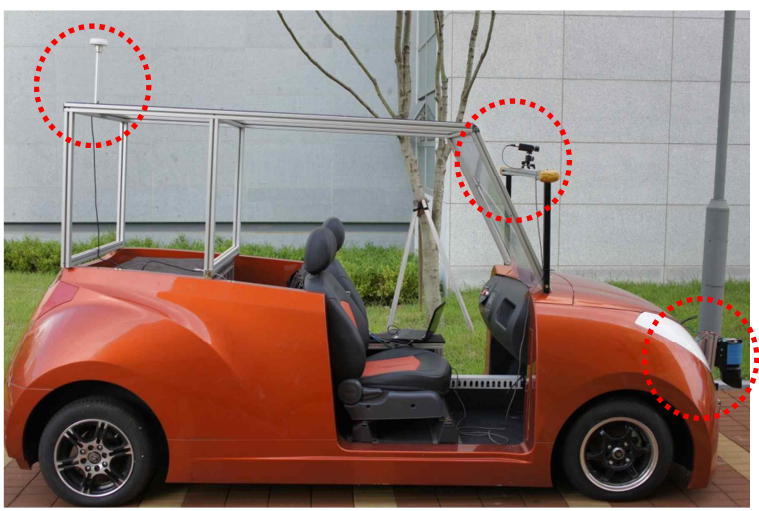

Figure 8: Sensors on SKKU UEV

the information received through PCU from each sensor is integrated to generate the path, which is then delivered to VCU to control the intelligent electric vehicle.

\subsubsection{Lane Detection and Keeping}

The lane is the most basic element among various features on the road, and it is detected using vision camera for unmanned autonomous driving. This report integrated two algorithms of image information to increase the lane detection rate. Lane information obtained by extracting lane edges through Canny filter and lane information detected from color information were used at the same time.
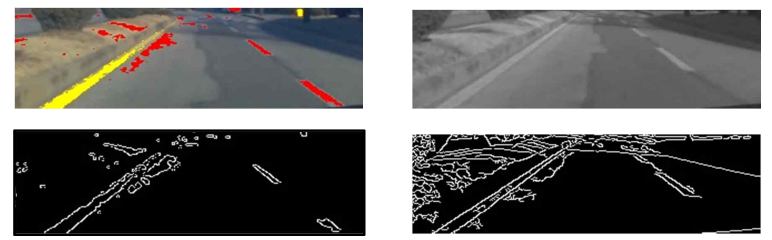

Figure 9: Pre-processing for Lane Detection

Then those images were applied with Hough transform to detect line components. Hough transform provides parameters of those line components $(r, \theta)$. After finding out valid data, lane can be de tected 


$$
r(\theta)=x_{0} \cos \theta+y_{0} \sin \theta
$$

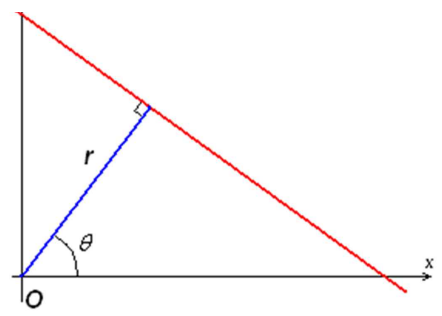

Figure 10: Hough Transform

\subsubsection{Obstacle Avoidance Algorithm}

It is necessary to develop an algorithm to detect and avoid obstacles such as road structures, other vehicles, or pedestrians. Laser scanner (LiDAR) can be used to detect an object in front of the vehicle and obtain distance $(l)$ and angle $(\theta)$ information. Distance information per $\Delta \theta$ section and obstacle existence probability density was made into a histogram. Using this information the vector field histogram (VFH) algorithm was applied for the obstacle avoidance.

SICK LiDAR is used to get the distance and angle of barriers and VFH algorithm was constructed using by LabVIEW Robotoics Module.

\subsubsection{Tracking Waypoint}

DGPS is used for the unmanned autonomous driving vehicle to find the destination. The target points are taken as waypoints and compared to current position, received from DGPS, for driving. DGPS module used in this study has error within $0.7 \mathrm{~m}$, and it is possible to make reception at the period of $1 \mathrm{~Hz}$. Also, Attitude and Heading Reference System (AHRS) sensor gives the direction of heading. Autonomous Driving algorithm has implemented by using the direction of heading and coordinates of waypoints and current position.

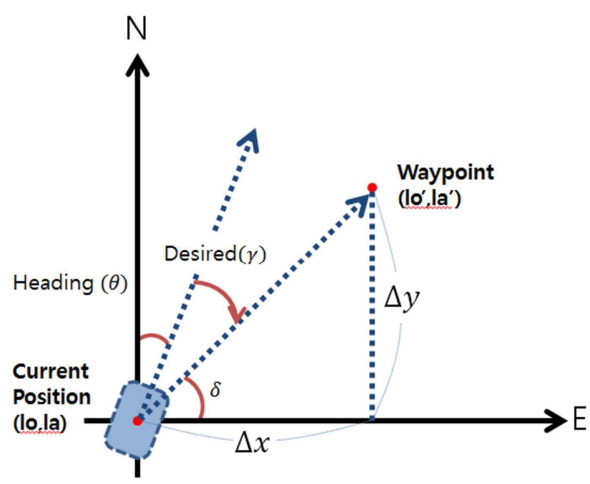

Figure 11: Waypoint Tracking
Longitude variation per $1 \mathrm{~m}: D_{o}=111.2 \mathrm{~km}$

Latitude variation per $1 \mathrm{~m}: D_{a}=\frac{2 \pi R_{c}}{360} \mathrm{~km}$

Equatorial radius: $6378 \mathrm{~km}$

Polar radius: $6350 \mathrm{~km}$

$$
\begin{aligned}
& R_{c}=\sqrt{\frac{\left(R_{e} R_{p}\right)^{2}}{R_{p}^{2}+R_{e}^{2} \tan ^{2} \theta}} \\
& \Delta x=\left(l^{\prime} a-l_{a}\right) \times D_{a} \\
& \Delta y=\left(l_{o}^{\prime}-l_{o}\right) \times D_{o} \\
& \text { Distance }=\sqrt{\Delta x^{2}+\Delta y^{2}} \\
& \delta=\tan ^{-1}\left(\frac{\Delta x}{\Delta y}\right)
\end{aligned}
$$

\section{Vehicle Test}

\subsection{Obstacle Avoidance Driving Test}

Obstacle avoidance driving test was performed. Some traffic corns were used as figure 12 . Obstacles were detected using by LiDAR, The vehicle avoided them by using Vector Field Histogram Algorithm.

Figure 14 shows how make a desired steering angle to moving into largest gap.

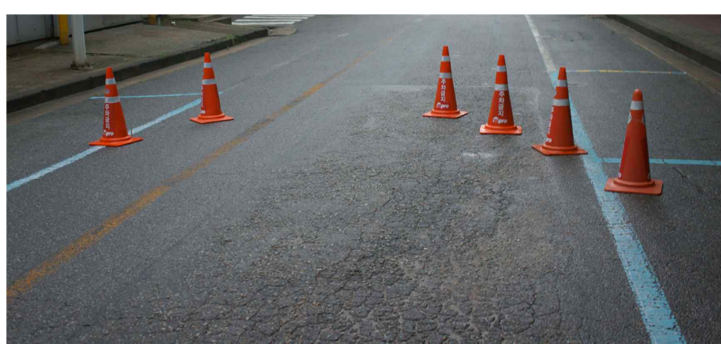

Figure 12: Obstacles using Traffic Corn

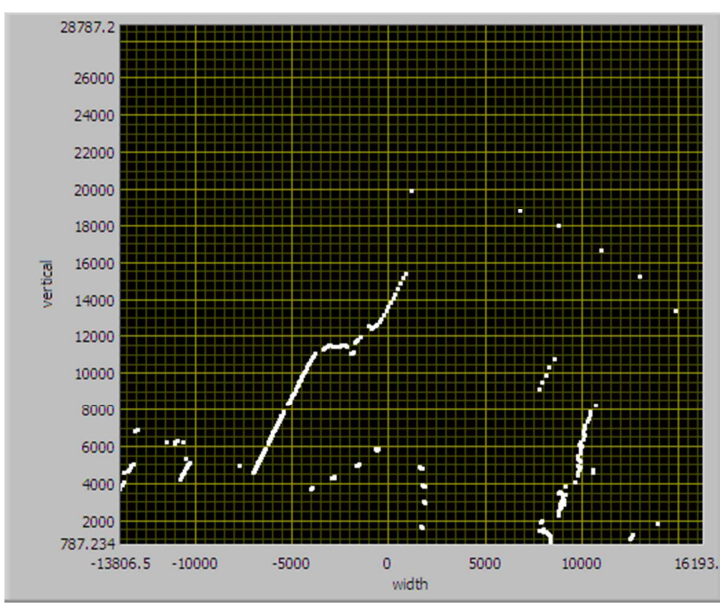

Figure 13: Raw data from LiDAR 


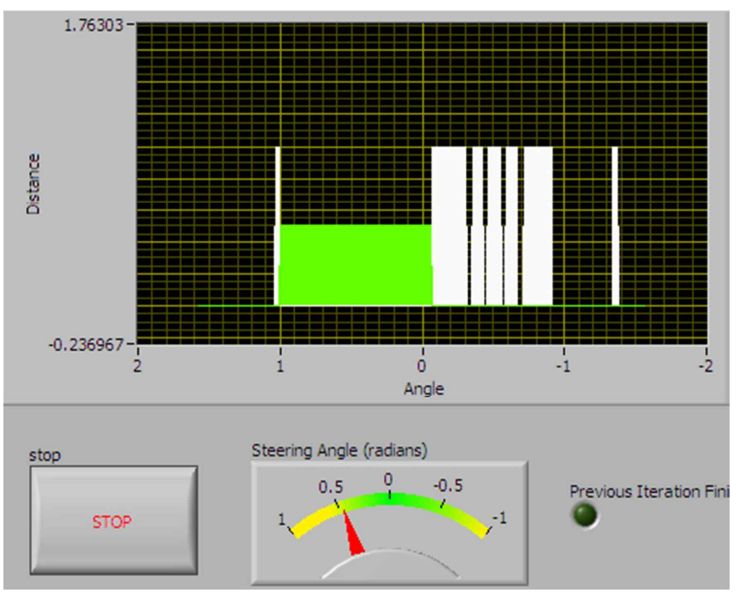

Figure 14: Vector Field Histogram and Desired Steering angle

\subsection{GPS Navigation Test with Lane Keeping System}

Autonomous driving test was performed; DGPS, AHRS, Camera was used to follow waypoints and keep the lane. The test was conducted $290 \mathrm{~m}$ on-road course, which contains straight and curve section, recording the distance in $8-10 \mathrm{~km} / \mathrm{h}$ speed.
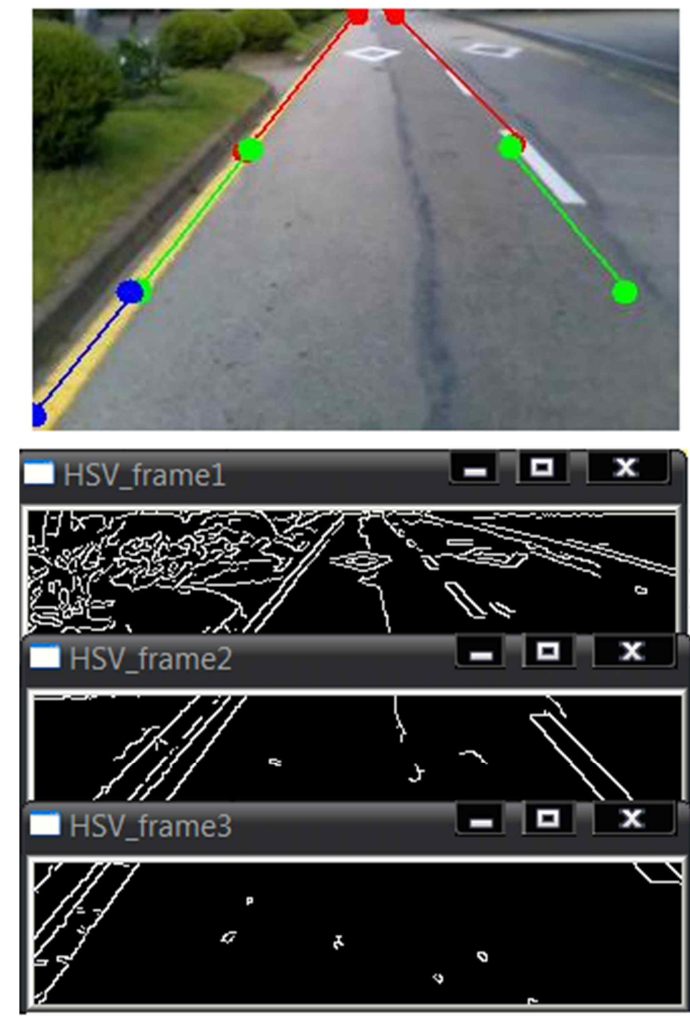

Figure 15: Lane Detection and Keeping

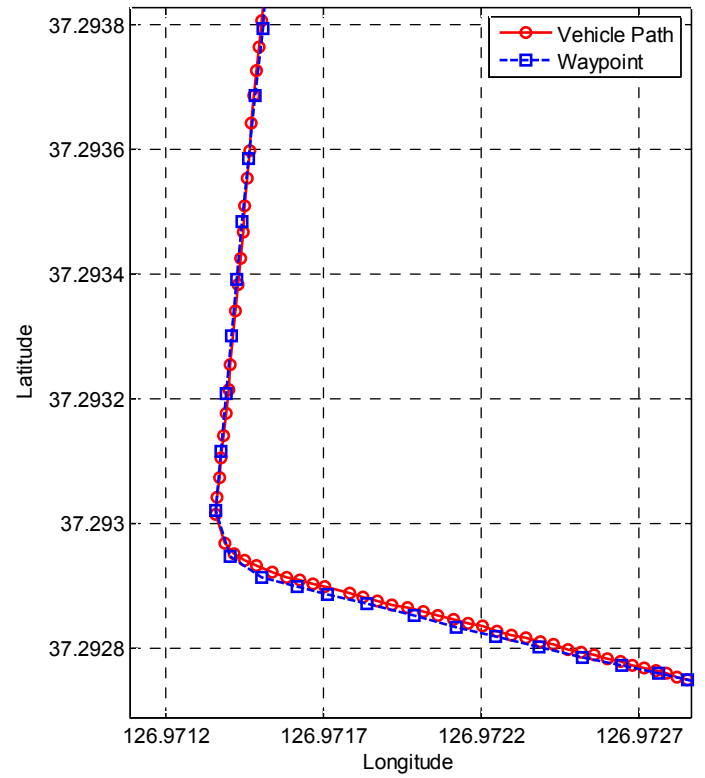

Figure 16: Vehicle Path and Waypoint

\section{Conclusion and Future Plans}

An intelligent electric vehicle was developed for development of unmanned autonomous driving algorithm. Also, through the developed intelligent electric vehicle platform, the performance of autonomous driving algorithm was evaluated and tested.

Through actual vehicle test, unmanned autonomous driving algorithms such as Lane Detection and Keeping, Obstacle Avoidance, Waypoint Tracking were verified, and it was found to be very effective in performing each independent algorithm.

Unlike unmanned vehicle which is remodeled an existing vehicle, it is possible to use the intelligent electric vehicle platform for application of more effective and diverse algorithms and sensors in construction of sensor, controller, and actuator.

In future, a stronger controller shall be designed to apply to actual road driving and not independent and limited circumstances, and cooperative control between algorithms and parallel computing will be used to implement integrative algorithm. 


\section{Referencing}

[1] J. S. Yun, B. Wang, S. H. Kim, S. H. Lee, S. H. Hwang, Development Of Cognitive Vehicle Platform For Neighborhood Electric Vehicle, KFPS, 2011, pp.297-304

[2] K. S. Yang, J. H. Park, I. Y. Kim, S. H. Hwang, Development of Path Plan Algorithm and Electric Vehicle Platform for Autonomous Driving, APICENS, 2013, pp.341-348

[3] J. Han, D. Kim, M. Lee, and M. Sunwoo, Enhanced Road Boundary and Obstacle Detection Using a Downward-Looking Lidar Sensor, IEEE Trans. Veh. Technol. Vol.61, No.3, 2012, pp.971-985

[4] S. Sukkarieh, E. M. Nebot, Hugh F. Durrent, A High Integrity IMU/GPS Navigation Loop for Autonomous Land Vehicle Applications, IEEE journals, 1999, pp.572-578

[5] J. Borenstein, Y. Koren, the Vector Field Histogram-Fast Obstacle Avoidance for Mobile Robots, IEEE Trans. Robotics Automat. Vol.7, No.3, 1991, pp.278-288

[6] C. Urmson and et al., Autonomous driving in urban environments: Boss and the Urban Challenge, Journal of Field Robotics, Vol. 25 , No. 8, 2008, pp. 425-466

[7] Azim Eskandarian, Handbook of Intelligent Vehicles, ISBN 978-0-85729-084-7, London, Springer, 2012

[8] IEEE News, http://www.ieee.org/about/news/2012/5sept ember 2 2012.html, accessed on 2013-0106

[9] Simple Vector Field Histogram VI, http://zone.ni.com/reference/enXX/help/372983B-01/lvrobovi/simple_vfh/, accessed on 2013-03-12

\section{Authors}

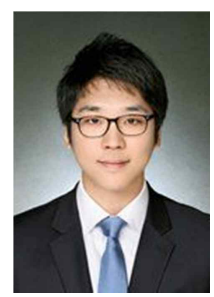

In Yub Kim received the B.S. degrees in electronic and electrical engineering from Sungkyunkwan University, Suwon, Korea, in 2012. He is currently working toward the M.S. degree with the School of Mechatronics Engineering, Sungkyunkwan University, Suwon, Korea. His research interests include the Driverless Vehicles and Electric Vehicles.
Kwan Suk Yang received the B.S. degrees in information and Communication engineering from Hansei University, Kunpo, Korea, in 2010 . He is currently working toward the M.S. degree with the School of Mechatronics Engineering, Sungkyunkwan University, Suwon, Korea. His research interests include the Autonomous Vehicles and Electric Vehicles.

Jin Joo Baek received the B.S. degrees in mechanical engineering from Daejin University, Pocheon, Korea, in 2012. She is currently working toward the M.S. degree with the School of Mechanical Engineering, Sungkyunkwan University, Suwon, Korea. Her research interests include Inwheel motor vehicle control

Sung-Ho Hwang received the B.S. degree in mechanical design and production engineering and the M.S. and Ph.D. degrees in mechanical engineering from Seoul National University, Seoul, Korea, in 1988, 1990, and 1997, respectively.

$\mathrm{He}$ is currently an Associate Professor with the School of Mechanical Engineering, Sungkyunkwan University, Suwon, Korea. 